\title{
Privacy Preserving Link Analysis on Dynamic Weighted Graph ${ }^{\star}$
}

\author{
Yitao Duan, Jingtao Wang, Matthew Kam, and John Canny \\ Computer Science Division \\ University of California, Berkeley \\ Berkeley, CA 94720, USA \\ \{duan, jingtaow, mattkam, jfc\}@cs.berkeley.edu
}

\begin{abstract}
Link analysis algorithms have been used successfully on hyperlinked data to identify authoritative documents and retrieve other information. They also showed great potential in many new areas such as counterterrorism and surveillance. Emergence of new applications and changes in existing ones created new opportunities, as well as difficulties, for them: (1) In many situations where link analysis is applicable, there may not be an explicit hyperlinked structure. (2) The system can be highly dynamic, resulting in constant update to the graph. It is often too expensive to rerun the algorithm for each update. (3) The application often relies heavily on client side logging and the information encoded in the graph can be very personal and sensitive. In this case privacy becomes a major concern. Existing link analysis algorithms, and their traditional implementations, are not adequate in face of these new challenges. In this paper we propose the use of weighted graph to define and/or augment a link structure. We present a generalized HITS algorithm that is suitable for running in a dynamic environment. The algorithm uses the idea of "lazy update" to amortize cost across multiple updates while still providing accurate ranking to users in the mean time. We prove the convergence of the new algorithm and evaluate its benefit using the Enron email dataset. Finally we devise a distributed implementation of the algorithm that preserves user privacy thus making it socially acceptable in real-world applications.
\end{abstract}

\section{Introduction}

Link analysis algorithms have been used successfully on hyperlinked data to identify authoritative documents and retrieve other information. For instance, the expertise location problem [1-4] is to find a person in an organization or community who is knowledgeable (and authoritative) in an area. Several approaches [1-3] construct an explicit social network between individuals, based on email or similar logs, and then use graphical analysis to locate the relevant experts. Similarly, the document ranking problem is to determine the relative levels of

\footnotetext{
* This material is based upon work supported by the National Science Foundation under Grant No. 0222745.
} 
"authoritativeness" among a collection of documents. Link analysis algorithms have been used in these environments to uncover such information $[5,6]$.

The primary drawback to the above approaches is the need for explicit structure about the social relations between individuals and the hyperlinks among documents, which do not necessarily exist. For instance, in a computer-mediated environment, a group of individuals could be using tools like software applications to access documents collaboratively, and there is neither an explicit social network representing how each individual is related to others, nor hyperlinks among documents. However, in such context, there are still compelling needs in identifying domain experts and authoritative documents.

Another inadequacy of such algorithms, when applied to authoritative document ranking, as Kleinberg acknowledged [5], is that they only make use of the structural information about the graph as defined by the links, and fail to capture patterns of user access which encode essential information about the user's attitude toward the document. The intuition behind the link analysis algorithms is that the link structure encodes important information about the nodes. For example, according to [5], the links among documents, be it hyperlinks on www or citations among academic papers, are constructed consciously by the authors of the documents and represent the authors' "endorsement" toward the authority of documents pointed to by the links and the HITS algorithm [5] can uncover such information to produce a ranking of documents according their level of authority. We believe that a similar principle also holds with patterns of user access: the way a user accesses a document could reflect his/her opinion about it. Meanwhile, a user's level of expertise can also be reflected by the documents that he/she accesses. There is a mutually reinforcing relationship between these two measures, which maps naturally to what Kleinberg denotes "hub" and "authority" [5]: a person is more likely to be an expert in an area if he/she reads more authoritative documents and a document is more likely to be authoritative if it is read by many experts. This phenomenon can also be observed in other graphs such as social networks where the structure is implicitly defined by communication.

In this paper we propose an approach to address these limitations and describe an algorithm that is suitable for such purpose. Notice that access pattern and link structure are not mutually exclusive. Rather, access pattern can complement or even define the other. Our approach uses weighted graphs to model the relationship among nodes and the weights can encode user access or communication. In situations where no explicit link structure exists, these weights effectively define the graphical structure and link analysis algorithms can be applied. Where there is an explicit link structure, weights obtained from access or communication analysis can be used to augment existing graph and uncover more information.

Using weights in identifying authoritative documents is not new [7]. The novelty of this paper is that we propose the use of weights to model user behavior and construct the link structure. This enables us to apply link analysis algorithms in settings where no such structure exists. However, computing on patterns of 
access or communication has two implications: (1) instead of a static system, the graph becomes dynamic. The model changes as more data is observed; and (2) user privacy becomes an issue due to the sensitive nature of the user's information used to construct and update the graph. (1) may also mandate that the system services users' query in real-time as there is no end to the accumulation of observations.

Meanwhile, emergence of new applications and changes of existing ones are making these problems more acute. For example, search engines are moving towards personalization (e.g. http://labs.google.com/personalized) that will be relying heavily on client side logging. While link analysis remains the core of their ranking algorithm (e.g. Google's PageRank [6]), the input data will be augmented by user configuration and access monitoring. Other applications in fields such as knowledge management, IR, HCI and data mining also showed similar trend.

To address these new issues, we devised Secure OnlineHITS, a distributed version and enhancement of Kleinberg HITS algorithm that (1) amortizes cost across a number of updates by using "lazy updates", which makes it more suitable for dynamic environments; and (2) uses cryptographic techniques to preserve user's privacy while performing the computation. Our technique can be easily extended to other link analysis algorithms such as Google's PageRank [6] but we won't pursue it in this paper. To make it concrete, we describe the algorithm in the context of document and expertise ranking. However, it is general enough to be applied to other situations where link analysis is appropriate. We use the term document in a broad sense. It refers to any information that can be identified, accessed and analyzed as a unit. For example, a web page or an image can all be classified as a document.

In the rest of the paper, we first review the original HITS algorithm in Section 2. We then discuss the construction of a weighted graph and prove the convergence of a HITS-like algorithm on such graphes in Section 3. In Section 4 we derive an online version of the HITS algorithm to make it more efficient to run in a dynamic environment on accumulated data. Evaluations are presented in Section 5. Finally in Section 6 we discuss privacy and security issues in running such kind of user activity analysis and describe our privacy preserving implementation of online HITS based on public-key encryption.

\section{A Review of HITS}

Kleinberg's HITS algorithm [5] is a well-known link analysis algorithm that identifies "authoritative" or "influential" webpages in a hyperlinked environment. Intuitively, by thinking of a hyperlink as a citation, a webpage $i$ is more of an authority (i.e. highly-referenced page) as compared to webpage $j$ if there are more hyperlinks entering $i$ from hub webpages, where a hub is simply a webpage that is a valuable source of links to other webpages. Likewise, a webpage $i$ is a better hub than webpage $j$ if there are more hyperlinks exiting $i$ into authoritative webpages. Given a set of $n$ webpages, HITS first constructs the 
corresponding $n$-by- $n$ adjacency matrix $A$, such that the element in row $i$ and column $j$ of A is 1 if there exists a hyperlink from webpage $i$ to webpage $j, 0$ otherwise. HITS then iterates the following equations:

$$
\begin{aligned}
& x^{(t+1)}=A^{T} y^{(t)}=\left(A^{T} A\right) x^{(t)} \\
& y^{(t+1)}=A x^{(t+1)}=\left(A A^{T}\right) y^{(t)}
\end{aligned}
$$

Where the $i$-th element of $x$ denotes the authoritativeness of webpage $i$ and the $i$-th element of $y$ denotes the value of webpage $i$ as a hub. With the vectors $x$ and $y$ initialized as vectors of ones and renormalized to unit length at every iteration, as $t$ approaches infinity, $x^{(t+1)}$ and $y^{(t+1)}$ approach $x^{*}$ and $y^{*}$, the principal eigenvectors of $A^{T} A$ and $A A^{T}$, respectively.

Even though HITS is originally intended to locate hubs and authorities in a hyperlinked environment, we observe that hubs and authorities map very well to the users and documents in access based link analysis and the relationship of mutual reinforcement still holds as mentioned in Section 1.

\section{Constructing a Weighted Graph}

By observing users behavior we can construct a graph of users/documents in environments where no such structure exists. We assume we can observe users' document access and communication pattern using tools like client side logger. Such tools are available from a number of sources. In particular, we have developed a version of our own that that can monitor user's document access and email communication. Of course such tools have serious privacy implications and we will address such issue in Section 6 .

The system consciously logs the users' activities as tuples of the form $\langle i, j\rangle$, which denotes the fact that user $i$ accesses document $j$ or communicates with user $j$, depending on the context. These log entries represent tacit data about the collaborative context because they do not directly encode the links between users nor documents. Given this activity log, we can construct a graph, such that vertices represent the users and/or documents and an edge $(i, j)$ exists and has non-negative weight $w_{i, j}$ iff an item $\langle i, j\rangle$ exists in the activity log.

How the weight $w_{i, j}$ is computed depends on the application and the goal of the link analysis. The ideas such as TFIDF [7], and the power law of practice [8], etc, are all good heuristics. In some situations the weight can be reduced to simple access or message count. This decision is orthogonal to our work and won't be pursued in this paper. The only assumption we make here is that $w_{i, j}$ is a non-negative, real number.

\subsection{Convergence of Weighted HITS}

Suppose we replace the $0-1$ valued element $A_{i j}$ in the adjacency matrix A with a non-negative weight function $w(i, j)$. First we introduce the following two lemmas from [9]. 
Lemma 1. If $M$ is a symmetric matrix and $v$ is a vector not orthogonal to the principal eigenvector $v^{*}$ of $M$, then the unit vector in the direction of $M^{k} v$ converges to $v^{*}$ as $k$ increases without bound.

Lemma 2. If a symmetric $M$ matrix has only non-negative elements, the principal eigenvector of $M$ has only non-negative entries.

According to the definition of $w(i, j)$, it's easy to see that matrix $A$ has only nonnegative values and the symmetric matrix $A^{T} A$ and $A A^{T}$ have only non-negative values, thus the principal eigenvectors of $A^{T} A$ and $A A^{T}$ have only non-negative entries (lemma 2).

In running HITS on a weighted graph, if we start with a vector $x$ with nonnegative entries, since $x$ is not orthogonal to the eigenvector of $A A^{T}$ which has only non-negative entries, the sequence $\left\{y^{(k)}\right\}$ converges to a limit $y^{*}$ (lemma 1 ). Similarly we can prove that the sequence $\left\{x^{(k)}\right\}$ converges to a limit $x^{*}$.

\section{Online HITS}

Access based graph construction and link analysis introduces a number of issues of its own such as frequent update, distributed data sources, data security and user privacy concerns, etc. An algorithm alone cannot address all these issues. But a properly designed algorithm can make addressing them a lot easier. In this section we describe a link analysis algorithm that works incrementally as data is being added. We use the idea of "lazy update" to avoid updating and running of the expensive computation so that we can amortize the cost across a number of updates while still maintaining enough precision.

\subsection{Basic Approach}

As shown in Section 1 and 3, the intuition behind HITS fits very well to our application. However, the algorithm is too expensive to run on every update, which can be quite frequent. Recall that the rankings we are seeking, $x$ and $y$, correspond to the principal eigenvectors of $A^{T} A$ and $A A^{T}$, respectively. A key observation is that a single update to the user access traffic corresponds to a perturbation to the $A$ matrix. Depending on the weight function selected, it can perturb a single element or a row of $A$. In either case the perturbation is local. This perturbation will cause variation to the principal eigenvector of $A^{T} A$ (and $A A^{T}$ ). If we can find the relationship between the variation of $x$ and $y$ and the perturbation to $A$, we can check each update to see if it will cause too much variation to $x$ and $y$. If the change is within acceptable tolerance, we can postpone applying the update thus avoiding running HITS for it. When the accumulated updates cause too much perturbation, we apply them together and run HITS once. This is essentially an approximation to HITS that amortizes its cost across multiple updates. We denote such an algorithm Online HITS. Another advantage of this approach is that service of user queries and updating $A$ and running of HITS can be made separate. The system can update $A$ and 
run HITS in background and continue servicing user queries with old results that we are confident to be within certain range from the latest ones. Users can enjoy nonblocking service.

Similar issues have been discussed in the context of stability of the HITS algorithm $[10,11]$. However, there is a subtle but significant difference between our approach and theirs: we are not concerned with the incompleteness of our data or the stability of the results. For us, the everlasting accumulation of data is an inherent feature of our system and the results we produce are the "best guess" based on the data we have so far. It is perfectly alright for the results to undergo dramatic change, which reflects the update of the system's knowledge about the world. Rather, we are interested in the bound of the change so that we can perform the tasks more efficiently. In addition, the conclusions in $[10$, 11] only apply to unweighted graphs represented by adjacency matrices. The theorem we describe below is applicable to any weighted graph.

Online HITS is based on the following theorem:

Theorem 1. Let $S=A^{T} A$ be a symmetric matrix. Let $a^{*}$ be the principal eigenvector and $\delta$ the eigengap ${ }^{1}$ of $S$. Let $E_{S}$ be a symmetric perturbation to $S$. We use $\|\cdot\|_{F}$ to denote the Frobenius norm ${ }^{2}$. For any $\epsilon>0$, if $\left\|E_{S}\right\|_{F} \leq$ $\min \left\{\frac{\epsilon \delta}{4+\sqrt{2} \epsilon}, \frac{\delta}{2 \sqrt{2}}\right\}$, then the principal eigenvector $\tilde{a}^{*}$ of the perturbed matrix $\overline{\tilde{S}}$ satisfies

$$
\left\|a^{*}-\tilde{a}^{*}\right\|_{2} \leq \epsilon
$$

This theorem gives us a way to test the perturbation and bound the error on the principal eigenvector. The proof is similar to that presented in [11] and is given in appendix.

There are two subtle issues that need to be addressed before we can use this theorem to construct an online HITS algorithm, namely the computations of eigengap $\delta$ and perturbation $\left\|E_{S}\right\|_{F}$. They have to be performed efficiently otherwise the cost of computing them would offset the saving of not running HITS. They will be addressed in the following subsections.

\subsection{Computation of Eigengap}

A straightforward way of computing eigengap $\delta$ is to calculate $\lambda_{1}$ and $\lambda_{2}$, the largest and the second largest eigenvalues, and take the difference. The original HITS algorithm is essentially a power method to compute the principal eigenvector of $S$. It can be revised easily, without adding complexity, to produce $\lambda_{1}$ and $\lambda_{2}$ as byproducts. Two modifications to the original HITS algorithm are introduced:

1. Instead of finding only the principal eigenvector, find the two eigenvectors corresponding to $\lambda_{1}$ and $\lambda_{2}$. This can be done by using the "block power

\footnotetext{
${ }^{1}$ Eigengap is defined to be the difference between the largest and the second largest eigenvalues.

2 The Frobenius norm of a matrix $X$ is defined by $\|X\|_{F}=\left(\sum_{i} \sum_{j}\left(X_{i j}\right)^{2}\right)^{1 / 2}$
} 
method" ([12], pp. 289). Concretely, start with two orthogonal vectors, multiply them all by $S$, then apply Gram-Schmidt to orthogonalize them. This is a single step. Iterate until they converge.

2. HITS normalizes the vector at each step to unit length. This is not necessarily the only choice to ensure convergence. Instead, we normalize each vector by dividing them by their first non-zero element. They still converge to the two eigenvectors and the scaling factors converge to $\lambda_{1}$ and $\lambda_{2}$ ([12], pp. 289).

The above modifications introduce extra computation of one eigenvector and Gram-Schmidt orthogonalization. The former doubles the work of HITS and the latter is $O(n)$. The total complexity is the same as HITS: $O(m n)$.

\subsection{Upper Bound of $\left\|E_{S}\right\|_{F}$}

Let $E$ be perturbation to matrix $A$ (This is our update to the graph). Then $\tilde{A}=A+E$ and $\tilde{S}=(A+E)^{T}(A+E)=A^{T} A+A^{T} E+E^{T} A+E^{T} E$. Let $E_{S}=A^{T} E+E^{T} A+E^{T} E$. We know for Frobenius norm (actually for any norms) $\|X+Y\|_{F} \leq\|X\|_{F}+\|Y\|_{F}$. So $\left\|E_{S}\right\|_{F} \leq 2\left\|A^{T} E\right\|_{F}+\left\|E^{T} E\right\|_{F}$. This bound involves matrix multiplication which we try to avoid. Note that the purpose of our online HITS is to postpone running the algorithm so that we can save some computation. This means that we will accumulate a number of updates (since the last time we update $A$ and run HITS). Even though each single update is local and involve only one element or one row of $A$, all the accumulated updates will affect a number of $A$ 's elements. This means $E$ can be sparse but unlikely to have only single non-zero element or a row. Let $E(t)$ be the accumulated unapplied update matrix after we observed $t$ th update (we reset the counting each time we apply updates). $E(t)=E(t-1)+\Delta(t)$ where $\Delta(t)$ has only one non-zero element or row. We have

$$
\left\|E_{S}(t)\right\|_{F} \leq 2\left\|A^{T} E(t)\right\|_{F}+\left\|E(t)^{T} E(t)\right\|_{F}
$$

where

$$
\begin{aligned}
\left\|A^{T} E(t)\right\|_{F} & =\left\|A^{T}(E(t-1)+\Delta(t))\right\|_{F} \\
& \leq\left\|A^{T} E(t-1)\right\|_{F}+\left\|A^{T} \Delta(t)\right\|_{F}
\end{aligned}
$$

and $\left\|E(t)^{T} E(t)\right\|_{F}=$

$$
\begin{aligned}
& \left\|(E(t-1)+\Delta(t))^{T}(E(t-1)+\Delta(t))\right\|_{F} \\
= & \| E(t-1)^{T} E(t-1)+E(t-1)^{T} \Delta(t) \\
& +\Delta(t)^{T} E(t-1)+\Delta(t)^{T} \Delta(t) \|_{F} \\
\leq & \left\|E(t-1)^{T} E(t-1)\right\|_{F} \\
& +2\left\|E(t-1)^{T} \Delta(t)\right\|_{F}+\left\|\Delta(t)^{T} \Delta(t)\right\|_{F}
\end{aligned}
$$

The three equations above give us a way to compute the upper bound on $\left\|E_{S}\right\|_{F}$ recursively. Namely we can keep running updates on the upper bounds of 
$\left\|A^{T} E(t-1)\right\|_{F}$ and $\left\|E(t-1)^{T} E(t-1)\right\|_{F}$ using Equation 4 and 5, respectively, and add to them the other terms in the equations to get new upper bounds for the next step.

When a single update involves only one element of $A, \Delta(t)$ has a single non-zero element. Let $\Delta_{i j}(t)$ be the non-zero element of $\Delta(t)$, then

$$
\begin{aligned}
\left\|A^{T} \Delta(t)\right\|_{F} & =\Delta_{i j}(t)\left\|A_{i *}\right\|_{2} \\
\text { and }\left\|E(t-1)^{T} \Delta(t)\right\|_{F} & =\Delta_{i j}(t)\left\|E(t-1)_{i *}\right\|_{2}
\end{aligned}
$$

where $A_{i *}$ and $E(t-1)_{i *}$ are the $i$ th row of $A$ and $E(t-1)$, respectively.

There are two ways to compute upper bounds of $\left\|A^{T} \Delta(t)\right\|_{F}$ and $\| E(t-$ $1)^{T} \Delta(t) \|_{F}$ : (1) keep the matrix $E(t-1)$ and use Equation $6 ;(2)$ use the largest elements of $A$ and $E(t-1)$ to estimate. (1) is accurate and involves $O(n)$ operations. (2) is fast (only scalar multiplication). The actual choice depends on application.

When an update changes a row of $A$, computing $\left\|A^{T} \Delta(t)\right\|_{F}$ and $\| E(t-$ $1)^{T} \Delta(t) \|_{F}$ is more expensive and requires $O\left(n^{2}\right)$ operations and $\left\|\Delta(t)^{T} \Delta(t)\right\|_{F}=$ $\left\|\Delta_{i *}(t)\right\|_{2}^{2}$ which is $O(n)$. This is at the same level of complexity as HITS but can be substantially cheaper to run because the latter takes a number of iterations to converge while the former needs to run only once. Kleinberg reported that the typical number of iterations for HITS to converge is 20 [5]. If the cost is still too high to accept, there are two ways to alleviate: (1) Frobenius norm has the property $\|A B\|_{F} \leq\|A\|_{F}\|B\|_{F} \cdot\left\|A^{T} \Delta(t)\right\|_{F}$ and $\left\|E(t-1)^{T} \Delta(t)\right\|_{F}$ can be reduced to scaler multiplication (with loss of "tightness"); (2) the computation can be made to be distributed across all clients, as described in Section 6 .

\subsection{The Algorithm}

Putting all these together, we summarize the Online HITS algorithm in this section. In the following, we assume there is a procedure Gram-Schmidt that, given a matrix $M$, orthogonalizes its column vectors using Gram-Schmidt process ([12], pp. 129). We also assume there is a process that monitors the data and invokes our algorithm with perturbation when it sees an update.

Let $z_{n}=(1,1, \ldots, 1)^{T} \in R^{n}$. Let $z_{n}^{\perp} \in R^{n}$ be the vector that is orthogonal to $z_{n}$ and has the same length. $\Delta \in R^{n \times m}$ is the perturbation caused by a single update. $\epsilon$ is the required precision. Let $x[1]$ be the first non-zero element of vector $x$. We keep global variables $\left\|E_{S}\right\|_{F},\left\|A^{T} E\right\|_{F}$ and $\left\|E^{T} E\right\|_{F}$. To make it concise, we use matrix computations in the pseudocode. However, it is clear that they can either be implemented together with HITS iterations, or only require operations on small number of the elements of the matrices involved, as described in Section 4.3.

The two main procedures are NewHITS and OnlineHITS. NewHITS is the modified version of HITS algorithm that performs block power iterations on two vectors and compute eigengap. Note that $A^{T} A$ and $A A^{T}$ share the nonezero eigenvalues so only one eigengap is needed. OnlineHITS is called on each 
update. It checks whether all the accumulated updates would cause large perturbation to the ranking. If so it will apply the updates and invoke NewHITS. Otherwise it returns the ranking from previous round. These two procedures are listed below.

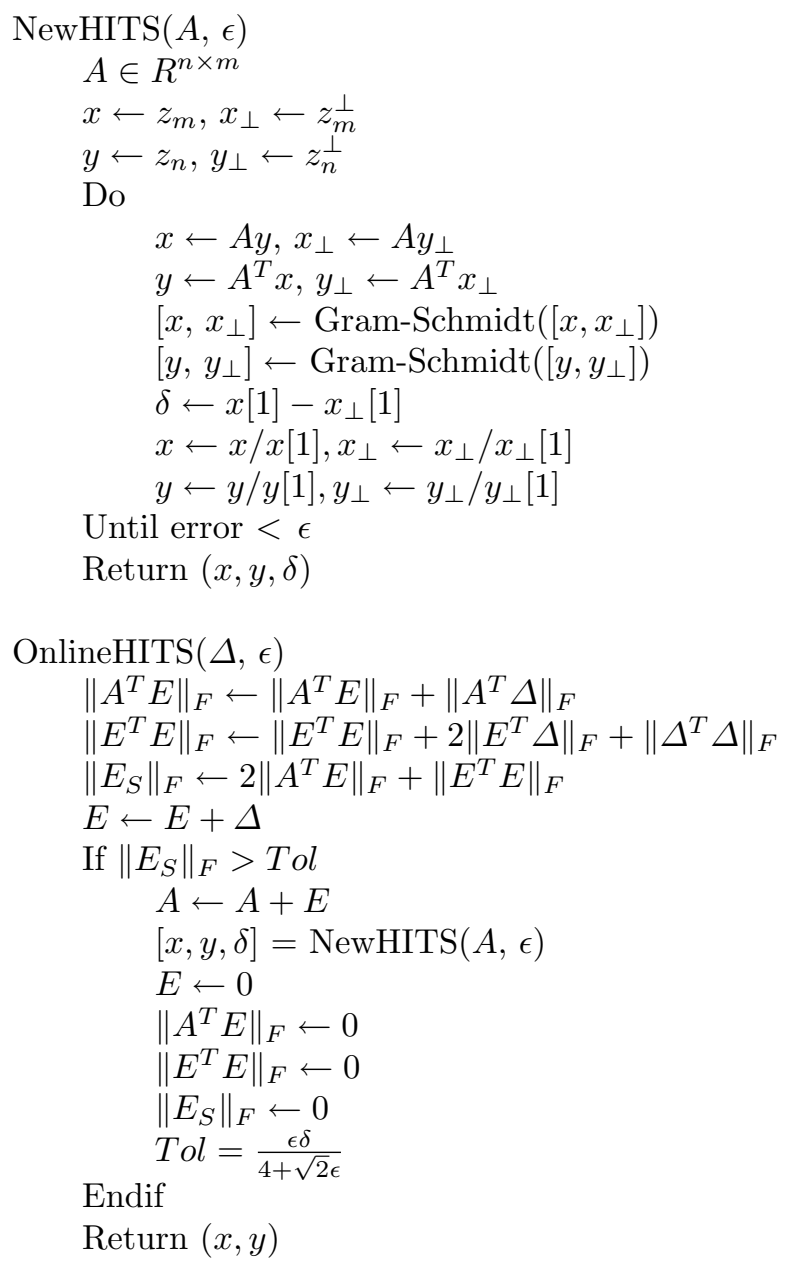

\section{Evaluation}

Compared to HITS, OnlineHITS is at the same complexity level. However, its advantage lies in the hope that the updates may not cause too much perturbation to the ranking so that recomputation is avoided. In addition, the operations introduced for perturbation checking do not require iteration so they are substantially cheaper than HITS. The benefit gained by Online HITS depends on the stability of the system in face of perturbation, which is application-specific. 
We believe that in situations where data is accumulating, Online HITS is most likely advantageous. The intuition behind this belief is that the more data is accumulated, the less significant a new update would be to the overall ranking. Therefore there would be more opportunities to avoid update and running of HITS.

To evaluate how well Online HITS performs, we implemented the algorithm and ran it on the Enron Email Dataset [13]. We used some of the useful mappings created by Andres Corrada-Emmanuel [14]. In particular, for each email, we used the mappings to find its author and recipients. As pointed out in [14], The Enron corpus contains some inconsistencies. In our test, we ignored emails that were mapped to multiple authors. Multiple recipients of a single email, however, are preserved.

This test can be thought of as "identifying the central figures" in the social network defined by the email communications. In constructing the graph, we simply used message count as the weight for each link between two users. Since one email may have multiple recipients, multiple links may be updated when an email is observed. There are a total of 150 users in the data set and our algorithm ranks them in "importance" according to their email communication.

An email is treated as a log item. Online HITS constantly monitors the log and performs operations as described in Section 4. A total of 8107 log items are observed. The precision is chosen to be $\epsilon=0.1^{3}$.

Note that we are not testing how well the ranking produced by Online HITS (or HITS) fits the "real" ranking which is a rather qualitative and subjective measure. Instead, we are examining Online HITS's algorithmic properties and how it performs more efficient than original HITS in a dynamic system.

The results of our test are shown in the following figures.

Figure 1 plots the ratio of the estimated upper bound of $\left\|E_{S}\right\|_{F}$ and its actual value. I.e. for each update $\gamma=\left(\left\|2 A^{T} E\right\|_{F}+\left\|E^{T} E\right\|_{F}\right) /\left\|E_{S}\right\|_{F}$. It shows how tight the upper bound given in Section 4.3 is. The number varies as updates accumulate and are applied, but never exceeds 3.8.

Figure 2(a) shows, for each update, the actual perturbation $\left\|E_{S}\right\|_{F}$, the upper bound we estimated based on the method of Section 4.3, and the tolerance as specified by Theorem 1 . Although the details are not easily discernable due to the large number of data points, it clearly shows the general trend of these measures, i.e. the tolerance grows as the data accumulates and allows for more and more perturbation while maintaining the given precision. Figure 2(b) enlarges one area of (a) to show the details. This area lies between data item 5965 to 6078 . The horizontal line segments of red dots represent the intervals where the perturbation is within tolerable range and no update is applied. This particular line in Figure 2(b) demonstrates around 113 updates for which the NewHITS needs

\footnotetext{
3 The choice of the precision depends on the application and the data. As we will observe later, the rankings of individual users in the Enron Email Dataset are quite "far" from each other and a larger $\epsilon$ can be used without affecting their relative standings. The result will be more saving in computation.
} 


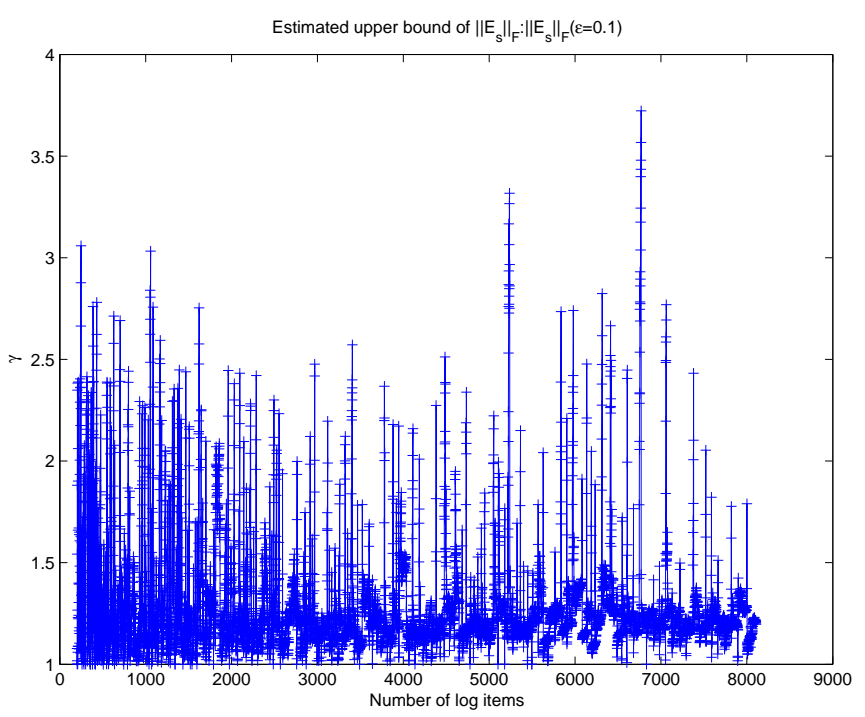

Fig. 1. Approximation ratio.
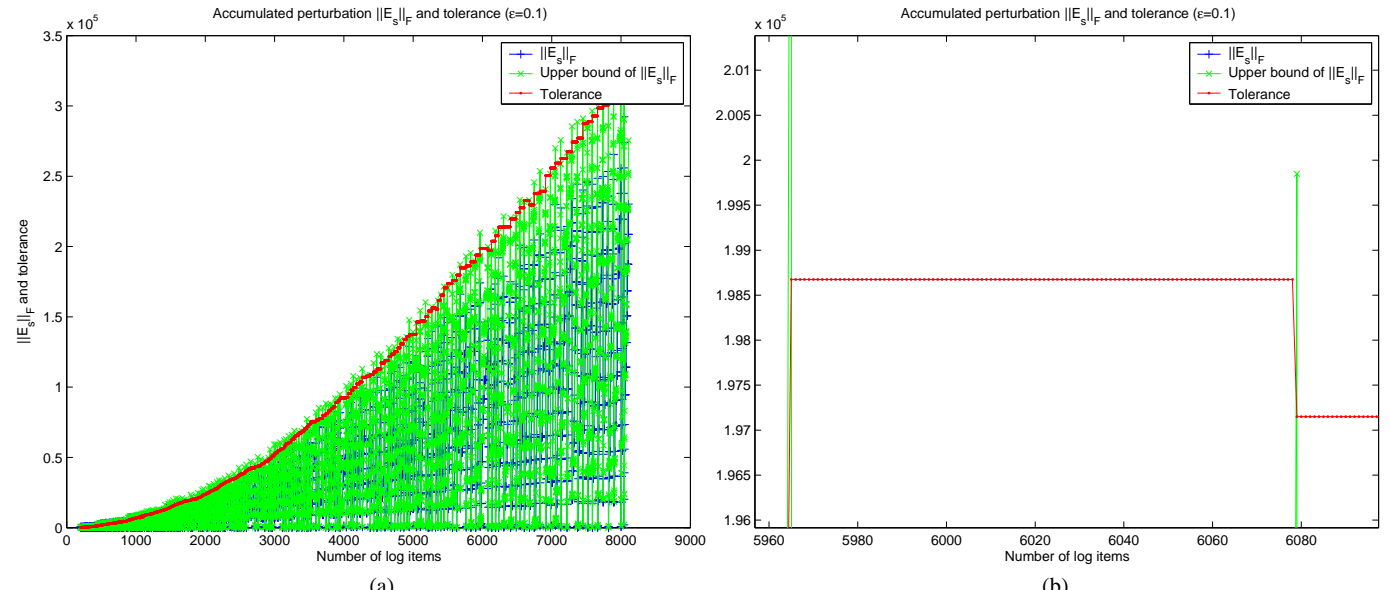

Fig. 2. Accumulated perturbation $\left\|E_{S}\right\|_{F}$ and tolerance. 
not to be invoked, i.e., a saving of 113 rounds of HITS computation. Similar savings can also be observed in other areas of Figure 2(a).
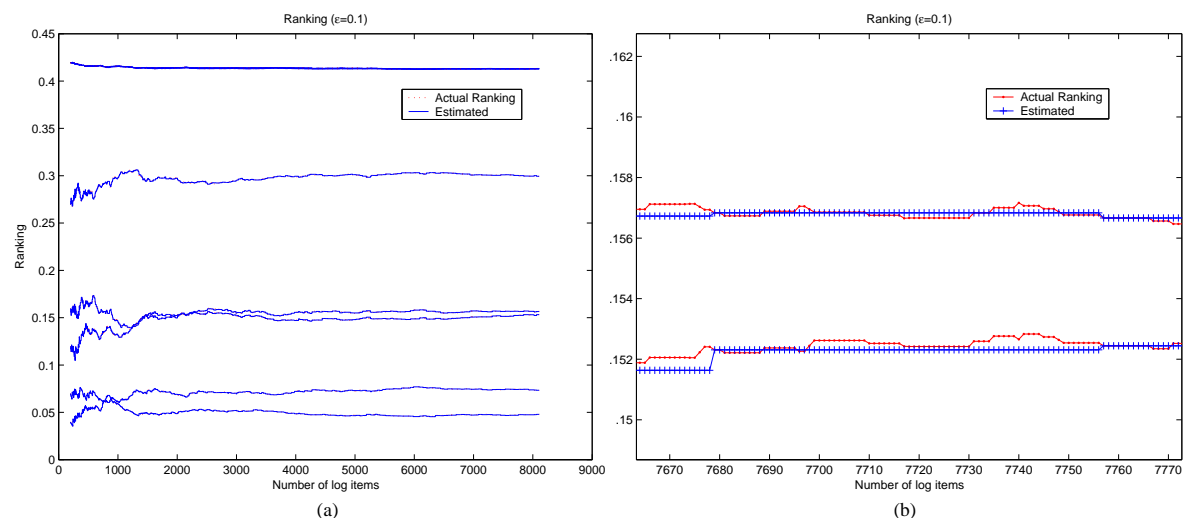

Fig. 3. Ranking.

Figure 3 shows the rankings of "top" 10 users in the data set ${ }^{4}$. Both the actual ranking of each user (obtained by running HITS at each update) and the approximation produced by OnlineHITS are plotted. Note that in Figure 3(a) the rankings of the top 5 users are so close that their results appear in the figure almost as a single curve (the curve on the top). Preliminary investigation uncovered that they are all involved in a large number of error messages (one of them is the sender and the rest recipients) and, as the HITS algorithm discovered, they share similar roles in terms of their email communication pattern in the data set. Our algorithm discovers this structure as well. The estimated rankings are so close to the actual ones that it is difficult to distinguish them in Figure 3(a). Figure 3(b) enlarges part of (a) for clarity. It shows that the estimated rankings closely track the actual ones even when no recomputation is performed.

Our test demonstrates the substantial advantage of OnlineHITS when applied to an actual data corpus. We believe it is applicable to other dynamic environments as well. In particular, for systems that do not have a clearly marked leisure period (e.g. a system serving users from all time zones around the world), simply "running HITS at night" will not work. Our algorithm can provide an accurate estimate on the perturbation a update can cause and offers precise ranking in real-time.

\section{$6 \quad$ Privacy Preserving Online HITS}

The algorithm described in previous sections addresses the dynamic and realtime response issues of using access patterns in link analysis. However, in many

\footnotetext{
${ }^{4}$ For privacy reasons the names of the users are withdrawn.
} 
situations, a naive implementation of the algorithm has severe privacy implications. In most applications, the weight on each edge of the graph represents the "rating" or "preference" of a user to the documents or other user and is gathered via client side logging. Such information is quite personal and exposing it would jeopardize the privacy of users thus hindering the acceptance of our system. If implemented directly, the online HITS algorithm would require the server running the algorithm be able to see all the data and involve substantial amount of network communication. In most situations trusting the server or network is not acceptable.

Fortunately this problem can be solved with cryptographic techniques. The general idea is that we can use encryption to protect user data and perform computations on encrypted data. Only aggregate data is made public and individual data are transmitted across the network in encrypted form. Such scheme has been proven to be sound and feasible, with satisfying performance. In [15], Canny proposed a privacy preserving collaborative filtering scheme that performs iterative SVD using the conjugate gradient method of Polak-Ribiere [16]. The basic building blocks, however, are homomorphism and threshold decryption which allow one to compute sums without disclosing summands. And this is exactly all that is needed to perform Online HITS. [15] gives complete description of the scheme and the proof of its soundness. Here we will sketch how it can be applied to make Online HITS distributed and privacy preserving.

In the following we will only consider the computation of document ranking, $x$. Expertise ranking is done in a similar fashion. We assume that most clients are honest and won't cheat or collude to pry about other's data. As in [15], we assume there is a write-once read-many (WORM) storage system where public data can be published. We also assume there is a tallier machine that performs addition on the data it receives. The tallier doesn't have to be a dedicated server. One of the clients can be designated as the tallier or its task can be made peerto-peer.

\subsection{Basics}

Several commonly used encryption schemes (e.g. RSA, Diffie-Hellman, ECC) have a useful property called homomorphism: if $m_{i}$ is a message and $E(\cdot)$ is an encryption function, let $g$ be a multiplicative group element, we can define a function $H(m)=E\left(g^{m}\right)$. This function satisfies

$$
H\left(\sum_{i=1}^{n} m_{i}\right)=\prod_{i=1}^{n} H\left(m_{i}\right)
$$

where multiplication is ring multiplication for RSA, or element-wise for DH or ECC. This allows one to obtain the encryption of a sum without revealing the summands.

Recovering the sum involves secret sharing and threshold decryption. The decryption key is not owned by any single party but secret-shared among all the clients. Pedersen's key generation protocol [17] or its variants/enhancements 
[18-20] can be used to securely generate the globally-known public key and distribute the secret shares of the private key among participants. At the end of their protocol, each client will have a share $s_{i}$ of the decryption key, $s$, which could have been easily reconstructed from any set $\Lambda$ of $t+1$ shares via Lagrange interpolation where $t$ is a pre-defined threshold that is greater than the maximum number of dishonest clients in the system. This arrangement not only discourages clients from cheating but also introduces redundancy that makes the system robust - any $t+1$ shares of $s$ can recover it. However, reconstructing $s$ will effectively reveal the secret key to a single party thus rendering the scheme useless. Instead, we use threshold decryption on the ciphertext when decryption is desired. That is, each client decrypts the ciphertext with its share of the key and the result is a share of the decryption of the value. By putting these shares together, users can recover the encrypted value.

The value decrypted is not actually the sum of messages $\sum_{i=1}^{n} m_{i}$ that we are seeking, rather it is $g^{\sum_{i=1}^{n} m_{i}}$. Although recovering the sum requires taking discrete log, the value of sum will be small enough $\left(10^{6}\right.$ to $\left.10^{9}\right)$ so that a babystep/giant-step approach is practical and the process can also be sped up by distributing it among many clients to be performed in parallel.

\subsection{A Run of HITS}

The results of the $t$ th iteration of HITS, $x^{t}$ and $y^{t}$ which are aggregate data, are made public. User $i$ is responsible for his own rating of the documents (obtained via analyzing his document access pattern), namely the $i$ th row of matrix $A$. Let $A_{i}^{T}=\left[a_{i 1}, a_{i 2}, \ldots, a_{i m}\right]$ be that row. For the step $x^{t+1}=A^{T} y^{t}$, all that is involved from $i$ is his own expertise ranking, $y_{i}^{t}$, and $A_{i}^{T}$. User $i$ computes $y_{i}^{t} A_{i}^{T}$ and encrypts the vector and sends it to the tallier. The tallier, after receiving data from all users, produces the encryption of the sums of the ranking of each document by multiplying corresponding elements of the vectors. The resulting vectors are committed to the WORM. Users will read from WORM and perform threshold decryption to recover the values. This is $x^{t+1}$.

To compute $y^{t+1}$ (which is $A x^{t+1}$ ), user $i$ computes $A_{i}^{T} x^{t+1}$ which is $y_{i}^{t+1}$, the $i$ th element of vector $y$ at iteration $t+1$, and publish it. If every user does this, the vector $y^{t+1}$ can be obtained.

The iteration can stop when enough precision is achieved.

\subsection{Perturbation Checking}

The scheme described in Section 6.2 shows a run of HITS, not Online HITS. To fit online HITS into such a scheme, we need to find a way to compute the perturbation, $\left\|E_{S}(t)\right\|_{F}$, with encrypted data or allow each user to compute with local data.

Recall that Equations 3, 4, 5 and 6 give us a way to update the upper bound of $\left\|E_{S}(t)\right\|_{F}$. The terms that need to be computed for each update are $\left\|A^{T} \Delta(t)\right\|_{F},\left\|E(t-1)^{T} \Delta(t)\right\|_{F}$ and $\left\|\Delta(t)^{T} \Delta(t)\right\|_{F}$. Since for user $i, \Delta(t)$ has non-zero elements only in its $i$ th row (and these numbers are obtained locally 
via his document access pattern analysis), $A^{T} \Delta(t)$ only involves the $i$ th row of $A$, which the user maintains. Similarly, $E(t-1)^{T} \Delta(t)$ only involves the $i$ th row of $E(t-1)$. In short, each user's update only involves his local data and it is straightforward to perform perturbation checking without disclosing private data: $\left\|E_{S}(t)\right\|_{F},\left\|A^{T} E(t)\right\|_{F}$ and $\left\|E(t)^{T} E(t)\right\|_{F}$ are made public via the WORM storage and each user will update them using Equations 4,5 and 6 with their local updates. When it is determined that it is time to update $A$, each user will update his own row and reset the perturbation records. All of them then collaboratively run the HITS algorithm as described in Section 6.2.

Note that we have actually killed two birds with one stone if we perform perturbation checking this way. Not only could we preserve user's privacy, we also distributed the computation among all users and parallelized the process.

There are some other issues in making such a scheme realistic such as dealing with dishonest users/tallier. Addressing them is out of the scope of this paper. [15] discusses such issues in detail and gives feasible solutions. In particular, it uses Zero Knowledge Proof (ZKP) to validate the data user and tallier produces so that they cannot excessively influence the results by cheating on their values.

\section{Related Work}

In [1], a set of heuristic graph algorithms are used to uncover shared-interest relationships among people, and to discover the individuals with particular interests and expertise, based on the logs of email communication between these individuals. The limitation with this approach is that experts are assumed to be communicating with fellow experts, which is not necessarily true in the realworld where experts may not be acquainted with one another, or may be rivals. Our approach does not assume any particular communication patterns between experts, and instead locate the experts based on their activities, e.g. if an expert accesses this set of authoritative documents, another person who accesses the same set is likely to be an expert as well.

The Referral Web $[2,3]$ is an interactive system for restructuring, visualizing and searching social networks on the World Wide Web. It constructs a graph of all users based on their email communication logs, which it uses to send a chain of referral requests until these requests reach an expert user. Like our Online HITS algorithm, Referral Web constructs the social network incrementally as it indexes the documents created and received by users. In contrast to our approach, however, the Referral Web raises possible privacy concerns because the chain of referrals inevitably reveal who someone down the chain knows to the user who initiates the search, unless individuals down the chain chooses not to forward the referral, in which case it becomes harder for the query to succeed.

Pirolli et al. [21] use a link-based approach like HITS to categorize webpages. It is similar to our weight-based algorithm in that users' access paths and metadata about webpages are used to construct the appropriate matrices. It differs significantly from ours in that while we use successive iterations to converge on our results, Pirolli et al. construct an activation network based on the strength of 
association between webpages and use the spread of activation in this network, starting from identified source webpages, to identify the webpages that exceed a threshold quantity of flow.

Carriere and Kazman's WebQuery system [22] rank webpages by considering the number of neighbors in the hyperlink structure that each webpage has. WebQuery performs link-based query post-processing to improve the quality of the results that it returns. In contrast, our incremental approach assumes that the hyperlink structure is highly dynamic, and postpones processing until the latest user-document accesses accumulate significant perturbation.

\section{Conclusion}

We extended the HITS hyperlink analysis algorithm to make it applicable to analyzing dynamic weighted graphs. Our generalizations are in two directions. First, we replaced the $0-1$ valued connectivity property with a non-negative valued weight function to allow for encoding of richer information. We proved the convergence HITS on such weighted graphes. Second, we created an online version of the HITS algorithm that can approximate the results efficiently in face of frequent updates by estimating the upper bound of perturbation and postponing applying the updates whenever possible. Both theoretical analysis and empirical experiments show that our generalized online algorithm is more efficient than the original HITS under the context of dynamic data.

Finally we developed a secure and distributed implementation of our online algorithm that solves the potential privacy issues that may occur when deploying large-scale access pattern-based document and authority ranking systems. Our scheme makes use of cryptographic techniques such as threshold decryption and homomorphic public-key encryption and distributes computation among users. Only aggregate or encrypted data are exposed. The scheme is also robust against a number of dishonest users up to a certain threshold.

Our extensions to Kleinberg's original HITS algorithm result in a generalized algorithm, Secure OnlineHITS, that is practical for link analysis in scenarios such as collaborative work and online communities, in which there is no explicit link structure among nodes, and that users' access patterns of documents are highly dynamic, complex and should remain private.

\section{References}

1. Schwartz, M.F., Wood, D.C.M.: Discovering shared interests using graph analysis. Comm. ACM 36 (1993) 78-89

2. Kautz, H., Selman, B., Milewski, A.: Agent amplified communication. In: AAAI96, Cambridge, Mass., MIT Press (1996) 3-9 Portland, Oreg.

3. Kautz, H., Selman, B., Shah, M.: Combining social networks and collaborative filtering. Comm. ACM 40 (1997) 63-65

4. MacDonald, D.W., Ackerman, M.S.: Just talk to me: A field study of expertise location. In: ACM CSCW-98. (1998) 315-324 
5. Kleinberg, J.M.: Authoritative sources in a hyperlinked environment. Journal of the ACM 46 (1999) 604-632

6. Brin, S., Page, L.: The anatomy of a large-scale hypertextual web search engine. In: 7th World-Wide Web Conference, Brisbane, Australia (1998)

7. Salton, G.: Automatic Text Processing: The Transformation, Analysis, and Retrieval of Information by Computer. Addison-Wesley (1989)

8. Newell, A., Rosenbloom, P.S.: Mechanisms of skill acquisition and the law of practice. In: J.R. Anderson (Ed.), Cognitive Skills and their Acquisition (pp. 155). Hillsdale, NJ: Earlbaum. (1981)

9. Golub, G., Loan, C.V.: Matrix Computations. Johns Hopkins University Press (1989)

10. Ng, A.Y., Zheng, A.X., Jordan, M.: Stable algorithms for link analysis. In: Proceedings of the 24th annual international ACM SIGIR conference on Research and development in information retrieval, ACM Press (2001) 258-266

11. Ng, A.Y., Zheng, A.X., Jordan, M.I.: Link analysis, eigenvectors and stability. In: Proceedings of the 17th International Joint Conference on Artificial Intelligence. (2001) 903-910

12. Strang, G.: Linear Algebra and Its Applications, 2nd Edition. Academic Press (1980)

13. Cohen, W.W.: Enron email dataset. (http://www-2.cs.cmu.edu/ enron/)

14. Corrada-Emmanuel, A.: Enron email dataset research. (http://ciir.cs.umass.edu/ ${ }^{\text {corrada/enron/) }}$

15. Canny, J.: Collaborative filtering with privacy. In: IEEE Symposium on Security and Privacy, Oakland, CA (2002) 45-57

16. Polak, E.: Computational Methods in Optimization. Academic Press (1971)

17. Pedersen, T.: A threshold cryptosystem without a trusted party. In: Proceedings of EUROCRYPT '91. Volume 547 of Springer-Verlag LNCS., Springer (1991) 522-526

18. Gennaro, R., Jarecki, S., Krawczyk, H., Rabin, T.: Secure distributed key generation for discrete-log based cryptosystems. Lecture Notes in Computer Science 1592 (1999) 295-310

19. Fouque, P.A., Stern, J.: One round threshold discrete-log key generation without private channels. Public Key Cryptography (2001) 300-316

20. Canny, J., Sorkin, S.: Practical large-scale distributed key generation. In: Eurocrypt 2004. (2004)

21. Pirolli, P., Pitkow, J., Rao, R.: Silk from a sow's ear: Extracting usable structures from the web. In: Proc. ACM Conf. Human Factors in Computing Systems, CHI, ACM Press (1996)

22. Carriere, J., Kazman, R.: Webquery: Searching and visualizing the web through connectivity. In: Proceedings of the International WWW Conference. (1997)

23. Stewart, G.W., Sun, J.G.: Matrix Perturbation Theory. Academic Press (1990)

\section{A Proof of Theorem 1}

Proof. We use ₹ to represent perturbed quantity. Suppose $S \in \mathbf{R}^{\mathbf{n} \times \mathbf{n}}$ is a symmetric matrix with principal eigenpair $\left(\lambda^{*}, a^{*}\right)$, and eigengap $\delta>0$. Let $E_{S}$ be a symmetric perturbation to $S$ such that $\tilde{S}=S+E_{S}$. By Theorem V.2.8 from matrix perturbation theory[23], there is some eigenpair of $\tilde{S}(\tilde{\lambda}, \tilde{a})$ such that

$$
\left\|a^{*}-\tilde{a}\right\|_{F} \leq \frac{4\left\|E_{S}\right\|_{F}}{\delta-\sqrt{2}\left\|E_{S}\right\|_{F}}
$$


and

$$
\left|\lambda^{*}-\tilde{\lambda}\right| \leq \sqrt{2}\left\|E_{S}\right\|_{F}
$$

assuming the denominator in 7 is positive. Let $L \in \mathbf{R}^{\mathbf{n}-\mathbf{1} \times \mathbf{n}-\mathbf{1}}$ be diagonal matrix containing all $S$ 's eigenvalues except $\lambda^{*}$. A bound similar to 8 holds:

$$
\|L-\tilde{L}\|_{F} \leq \sqrt{2}\left\|E_{S}\right\|_{F}
$$

Let $\tilde{\lambda_{2}}$ be the largest eigenvalue in $\tilde{L}$. By Corollary IV.3.6 of [23], Equation 9 implies

$$
\tilde{\lambda_{2}} \leq \lambda_{2}+\sqrt{2}\left\|E_{S}\right\|_{F}
$$

Since $\left\|E_{S}\right\|_{F} \leq \frac{\delta}{2 \sqrt{2}}$, Equations 8 and 10 ensures that $\tilde{\lambda}>\tilde{\lambda_{2}}$, i.e. $(\tilde{\lambda}, \tilde{a})$ is indeed the principal eigenpair of $\tilde{S}$. Also this will ensure the denominator in 7 is indeed positive.

Given any $\epsilon>0$, if $\left\|E_{S}\right\|_{F} \leq \frac{\epsilon \delta}{4+\sqrt{2} \epsilon}$, then $\frac{4\left\|E_{S}\right\|_{F}}{\delta-\sqrt{2}\left\|E_{S}\right\|_{F}} \leq \epsilon$ thus we have $\left\|a^{*}-\tilde{a}\right\|_{2} \leq \epsilon$. 